\title{
SIMULINK BASED DESIGN SIMULATIONS OF BAND PASS FIR FILTER
}

\author{
Lalit Maurya $^{1}$, Vijay Kr. Srivastava ${ }^{2}$, Rajesh Mehra ${ }^{3}$ \\ ${ }^{1,2}$ M.E. Student, ${ }^{3}$ Associate Professor Electronics and Communication, National Institute of Technical Teacher \\ Trainingand Research, Punjab University, Chandigarh
}

\begin{abstract}
In this paper, window function method is used to design digital filters. The Band Pass filter has been design with help of Simulink in $M A T L A B$, which have better characteristics of devising filter in fast and effective way. The band pass filter has been design and simulated using Kaiser window technique. This model is established by using Simulink in MATLAB and the filtered waveforms are observed by spectrum scope to analyze the performance of the filter.
\end{abstract}

Keywords: FIR, window function method, Kaiser, Simulink, MATLAB.

$* * *$

\section{INTRODUCTION}

Digital filter is a type of digital system that filters discretetime signal and main objective performing frequency domain filtering by processing sample data. It can be divided into two types in accordance with the time domain features of unit impulse response function: Infinite impulse response (IIR) and finite impulse response (FIR).Compared with IIR filter, FIR filter can have exactly linear phase characteristic while satisfying amplitude frequency response. The implication of this is that no phase distortion is introduced into the signal by filter [1]. Therefore, it is widely used in high-fidelity signal processing, such as digital audio, image processing, data transmission, biomedicine and other fields. SIMULINK is a software package in MATLAB which is specially used for dynamic system modeling, simulation and analysis. We can see the simulation result in a virtual way by using Simulink built in MATLAB that how the filter specification meet to perform the filtering operation [2].

\section{FILTER STRUCTURE}

Assuming the unit impulse response $\mathrm{h}(\mathrm{n})$ is a sequence of $\mathrm{N}$ points of the filter, $0 \leq \mathrm{n} \leq \mathrm{N}-1$, thus the system function of the filter is expressed as follows:

$$
H(z)=\sum_{n=0}^{N-1} h(n) z^{-n}
$$

The function above can be expressed as a difference equation:

$$
y(n)=\sum_{m=0}^{N-1} h(m) x(n-m)
$$

Obviously, equation (2) is a convolution sum formula of linear shift-invariant system as well as a horizontal structure of the delay chain of $\mathrm{x}(\mathrm{n})$, thus FIR horizontal structure is as follows:

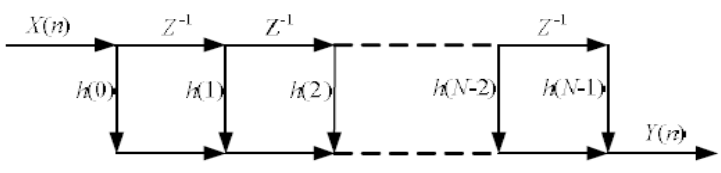

Fig 1 Direct- form Structure

Structures in which the multiplier coefficients are precisely the coefficients of the transfer function are called direct form structures. Noting that $\mathrm{N}$ represents tap numbers of the FIR filter; $x(n)$ is for the input sample of nth time; $h(m)$ acts as the mlevel tap coefficients of the FIR filter [3].

A higher - order FIR transfer function can also be realized as a cascade of FIR section with each section describe by either a first-order or a second-order transfer function. Now the system function $\mathrm{H}(\mathrm{z})$ can be decomposed into second-order real coefficients factor form as show in (3):

$H(z)=\sum_{n=0}^{N-1} h(n) z^{-n}=\prod_{i=1}^{\frac{N}{2}}\left(\beta_{0 i}+\beta_{1 i} z^{-1}+\beta_{2 i} z^{-2}\right)$

Its structure form as follows:

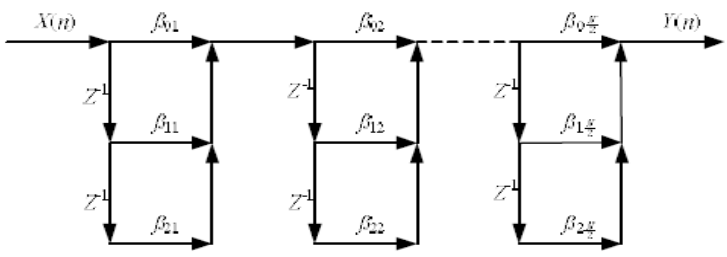

Fig 2 Cascade Structure 
Conjugate poles are controlled by every joint among the structure for conveniently transmitting zeros. This type of structure requires more coefficients and multiplication than the direct one so it is not often used[4].

We are familiar with that if unit impulse response function $h(n)$ of FIR filter is real and satisfies the following conditions:

$$
\left\{\begin{array}{c}
h(n)=h(N-1-n) \\
h(n)=-h(N-1-n)
\end{array}\right.
$$

From the above equation it can be realized that the center of the symmetry has the coordinate of $(\mathrm{N}-1) / 2$ which shows that the filter is linear phase. [5]. The symmetrical characteristic of the FIR filter can simplify network structure (here only for discussion when $\mathrm{h}(\mathrm{n})$ is an even-symmetry case and $\mathrm{N}$ is an even number. It's the same theory when $h(n)$ is an oddsymmetry presentation) when $\mathrm{h}(\mathrm{n})$ is even symmetry and $\mathrm{N}$ is an even number:

If a given unit impulse response of linear FIR filter is $h(n), 0$ $\leq \mathrm{n} \leq \mathrm{N}-1$, and it satisfies either of the symmetrical conditions as represented in (4).

When $\mathrm{h}(\mathrm{n})$ is for the dual symmetry, $\mathrm{N}$ is an even number:

$$
\begin{aligned}
H(z)=\sum_{n=0}^{N-1} h(n) z^{-n} & \\
& =\sum_{n=0}^{(N / 2)-1} h(n) z^{-n}+\sum_{n=N / 2}^{N-1} h(n) z^{-n}
\end{aligned}
$$

Among the second $\sum$, we put $n=N-1-m$, and change $m$ with $\mathrm{n}$ to get the expression:

$$
\begin{aligned}
H(z)=\sum_{n=0}^{(N / 2)-1} h(n) z^{-n} & \\
& +\sum_{n=0}^{(N / 2)-1} h(N-1-n) z^{-(N-1-n)}
\end{aligned}
$$

On the basis of linear parity- symmetry conditions: $h(n)=$ $\pm \mathrm{h}(\mathrm{N}-1-\mathrm{n})$, with (6) it modified into new formula as follows:

$$
H(z)=\sum_{n=0}^{\left(\frac{N}{2}\right)-1} h(n)\left[z^{-n} \pm z^{-(N-1-n)}\right]
$$

Its linear network structure is presented as following picture:

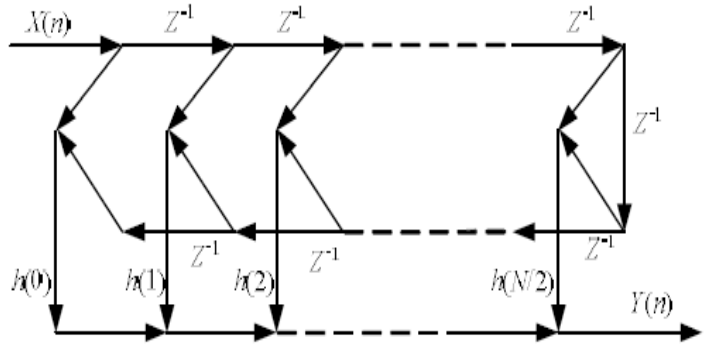

Fig 3 h(n) for the dual symmetry, $\mathrm{N}$ is an even

When $\mathrm{h}(\mathrm{n})$ is for the dual symmetry, $\mathrm{N}$ is an odd number, its linear network structure is presented as follows [6]:

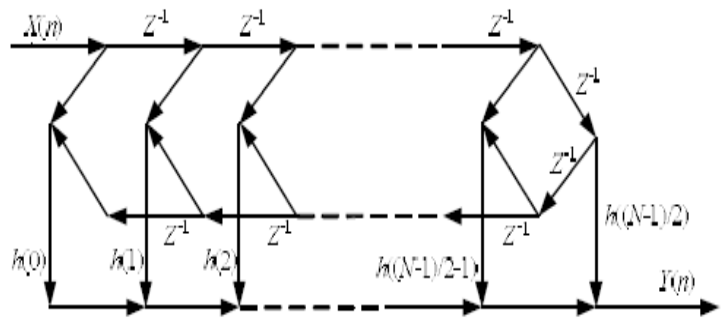

Fig $4 \mathrm{~h}(\mathrm{n})$ for the dual symmetry, $\mathrm{N}$ is an odd

From the above analysis we conclude that: linear phase FIR filter require half of multiplications compared with a directform one. However, we choose the direct form due to its design convenience and the same linear phase restrictions will be satisfied when it is consistent with linear restrictions [7].

\section{WINDOW FUNCTION}

The most straightforward approach to obtain a causal finite impulse response (FIR) is to truncate and smooth the ideal response. We begin with the desired frequency response specification $\operatorname{Hd}\left(\mathrm{e}^{\mathrm{j} \omega}\right)$ and determine its unit sample responsehd(n). Indeed, $\operatorname{hd}(n)$ is related to $\operatorname{Hd}\left(\mathrm{e}^{\mathrm{j} \omega}\right)$ by the Fourier Transform relation:

$$
H\left(e^{j \omega}\right)=\sum_{n=0}^{N-1} h(n) e^{-j \omega n}
$$

Where

$$
h_{d}(n)=\frac{1}{2 \pi} \int_{-\pi}^{\pi} H_{d}\left(e^{j \omega}\right) e^{j \omega n} d \omega
$$

In general, the unit sample response hd(n) obtained from (9) is infinite in duration and must be truncated at some point to devise an FIR filter of length $M$. One effective way for truncation of $\mathrm{hd}(\mathrm{n})$ to some finite length is equivalent to multiplying hd(n) by a length-finite window sequence namely: $h(n)=\omega(n) h d(n)$, and turning noncasual sequence into a casual one[8]. 
Frequently-used window functions can be listed as Triangular windows, Hann window, Hamming window, Kaiser windows and Blackman windows and so forth. All kinds of functional parameters list is as follows:

TABLE I. PARAMETERS TABLE OF VARIOUS WINDOW FUNCTIONS

\begin{tabular}{|l|c|c|c|}
\hline $\begin{array}{c}\text { Window } \\
\text { function }\end{array}$ & $\begin{array}{c}\text { Over band } \\
\text { width } \\
(\mathrm{P} / \mathrm{N})\end{array}$ & $\begin{array}{c}\text { Minimum } \\
\text { stopband } \\
\text { attenuation } \\
(\mathrm{dB})\end{array}$ & $\begin{array}{c}\text { Sidelobe peak } \\
\text { amplitude } \\
(\mathrm{dB})\end{array}$ \\
\hline Rectangle & 4 & 21 & 13 \\
\hline Triangle & 8 & 25 & 25 \\
\hline Hann & 8 & 44 & 31 \\
\hline Hamming & 8 & 53 & 41 \\
\hline $\begin{array}{c}\text { Kaiser } \\
(\beta=5.6)\end{array}$ & 7.442 & 60 & 51 \\
\hline Blackman & 12 & 74 & 71 \\
\hline
\end{tabular}

The kaiser window function don't have fixed characteristics, such as transition width and stopband attenuation. This type of problem is overcome by incorporating a ripple control parameter, $\beta$, which allows the designer to trade-off the transition width against ripple [9]. In this paper the kaiser window function is used. The parameters of kaiser window is as follows:

Normalized transition zone:

$$
\Delta \mathrm{f}=\frac{\omega_{\mathrm{s}}-\omega_{\mathrm{p}}}{2 \pi}
$$

$\omega p$ represents the passband cutoff frequency, $\omega$ s represents the stopband cutoff frequency.

The order of the filter

$$
M=\frac{A_{s}-7.95}{14.36 \Delta f}
$$

As is the minimum stopband attenuation.

When As is separately defined as:

As $<21,21<$ As $<50$, As $<50$. We can obtain values of $\beta$ correspondently as follows:

$$
\left\{\begin{array}{c}
\beta=0 \\
\beta=0.584\left(\mathrm{~A}_{\mathrm{s}}-21\right)^{0.4}+0.07886\left(\mathrm{~A}_{\mathrm{s}}-21\right) \\
\beta=0.1102\left(\mathrm{~A}_{\mathrm{s}}-8.7\right)
\end{array}\right.
$$

The performance of various window functions has been listed in the table and the appropriate window function can be selected according to the parameters in order to accomplish a given design [10].

The design steps for the window function as follows:
1) Given the desired frequency response functionHd $\left(\mathrm{e}^{\mathrm{j} \omega}\right)$.

2) Calculating hd $(\mathrm{n})=\operatorname{IDTFT}\left[\operatorname{Hd}\left(\mathrm{e}^{\mathrm{j} \omega}\right)\right]$.

3) Judging by the chart tableI, The shape of the window and the size of $\mathrm{N}$ should be certain due to the requirements of the transition bandwidth and the minimum stopband attenuation.

4) Determine the unit sampling response of the FIR design $\mathrm{h}(\mathrm{n}), \mathrm{n}=0,1, \ldots \mathrm{N}-1$

5) Calculating $\left.\operatorname{Hd}\left(\mathrm{e}^{\mathrm{j} \omega}\right)\right)$ = DTFT [hd(n)], and verifying if it meets the requirements otherwise redesign it[11].

In short, window function method is most simple and effective way as well as the most common way for designing FIR filter.

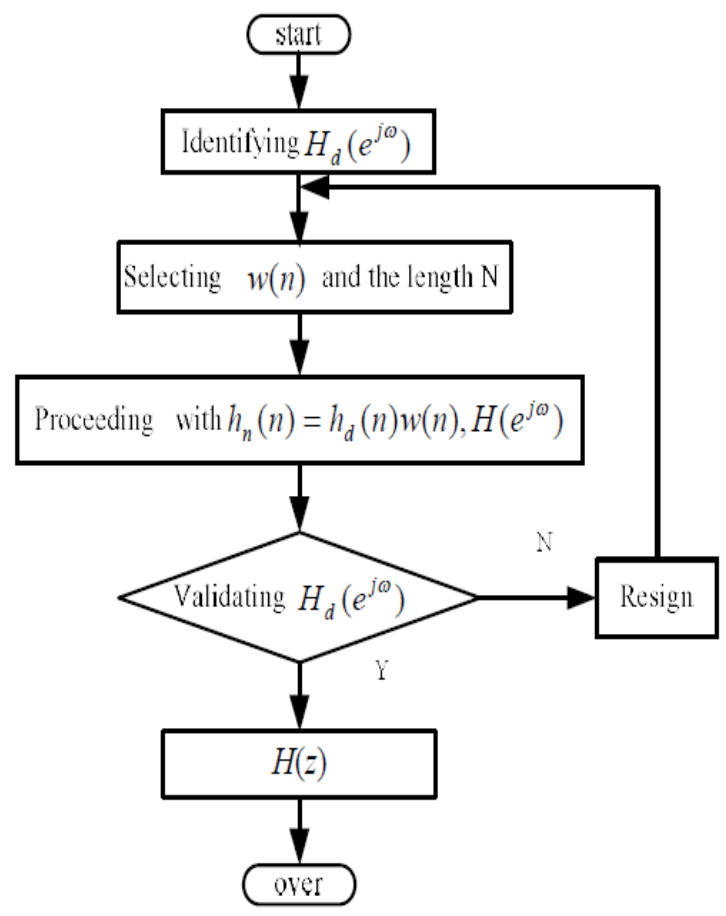

Fig 5.Design flow of filter design by window function

\section{SIMULINK BASED DESIGN SIMULATION}

In this paper we will apply a sinusoidal wave which is a mixed signal of $100 \mathrm{~Hz}, 250 \mathrm{~Hz}$ and $400 \mathrm{~Hz}$ frequencies to FIR band pass filter which is design by the Simulink in MATLAB. The Required Parameters for designing the filter are $\mathrm{Fs}=1 \mathrm{KHz}$, lower cut-off frequency fc1 $=150 \mathrm{~Hz}$, upper cut-off frequency of passband $\mathrm{fc} 2=350 \mathrm{~Hz}$ to fulfill Kaiser Window.

The simulation model on Simulink in MATLAB for filters output observation on the application of given sinusoidal wave is as follows [12]. 


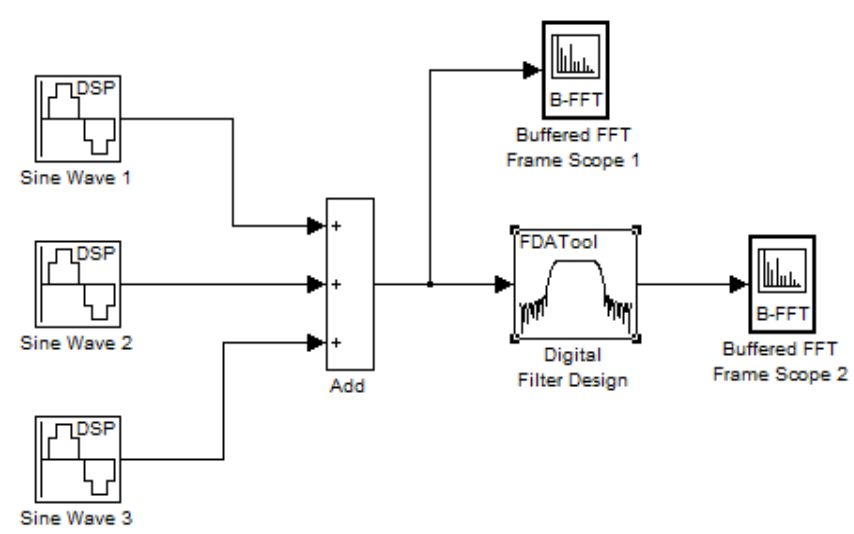

Fig 6: Simulink Simulation Model

The Magnitude response of FIR filter which is design in Simulink with Kaiser window function of specified order $\mathrm{n}=$ 38 and beta $=3.5$ as follows

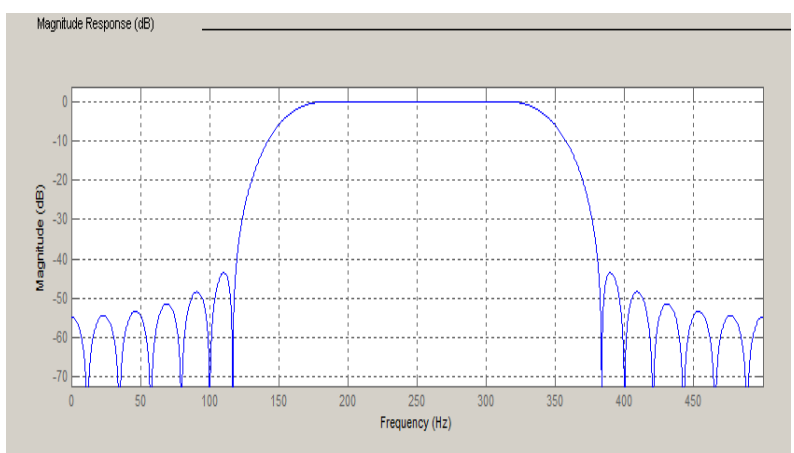

Fig: 7 Magnitude response of specified order filter

The spectral response for the given mixed sinusoidal signal which is applied at the input of the filter is shown in figure 8 and spectral response of the output of the filter is shown in figure 9 .

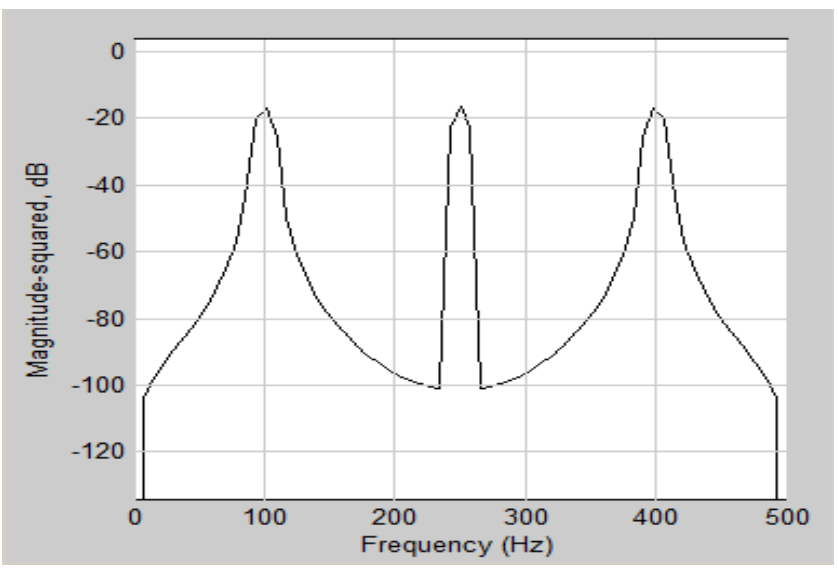

Fig 8: Spectral response of mixed sinusoidal signal

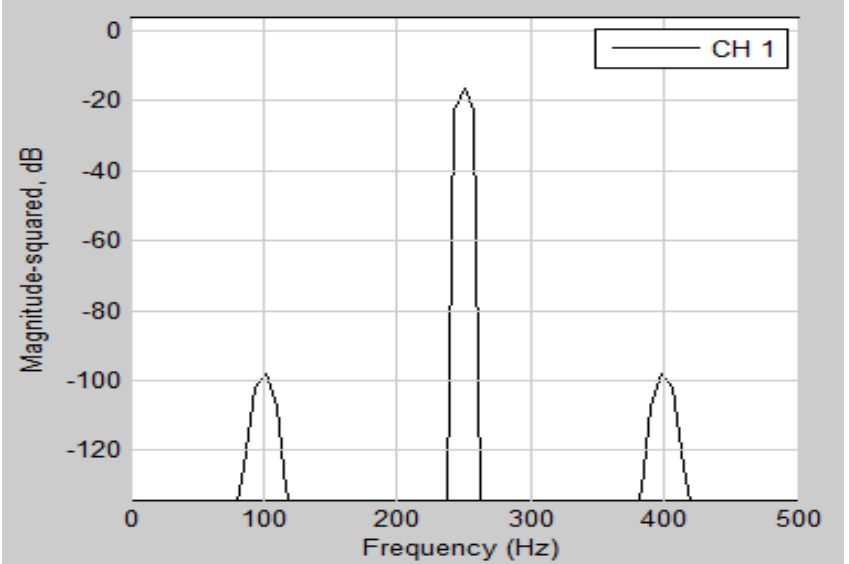

Fig 9: Spectral response of the output of the filter

Further we can use minimum order FIR filter which is itself provided by the FDAtool with Kaiser window function to filter to mixed signal and magnitude response of design filter and output spectrum shown in figure 10 an 11.

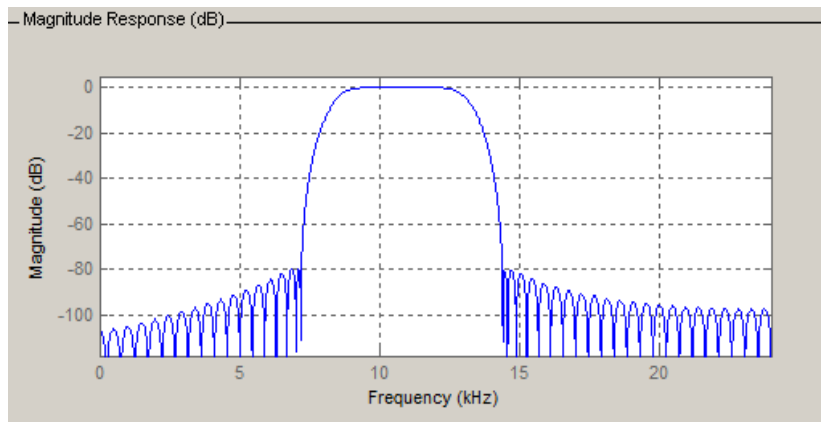

Fig 10: Magnitude response of minimum order kaiser window filter (generated itself by FDAtool)

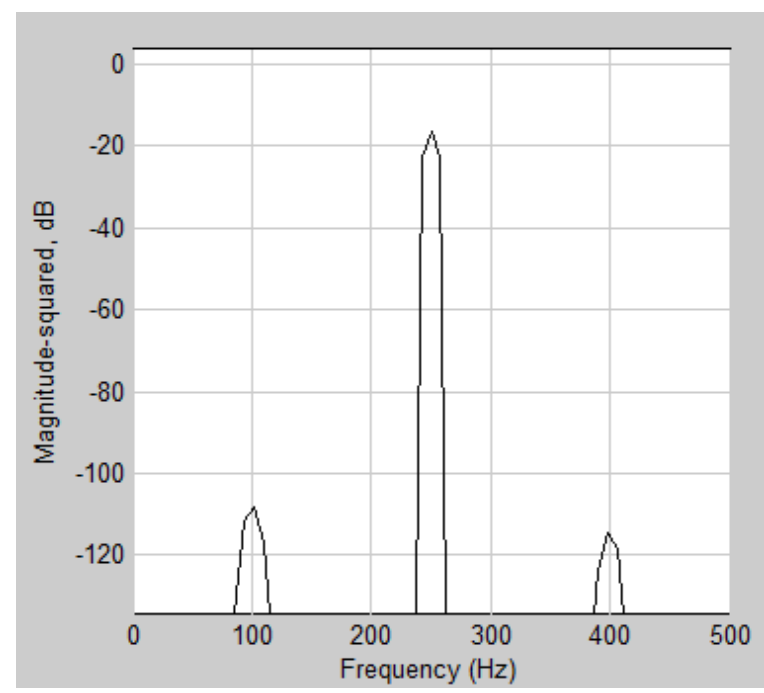

Fig 11: output spectrum of minimum order filter 


\section{CONCLUSIONS}

Window function method is used to achieve the finite impulse response with the help of window function with certain width to intercept an infinite impulse response. We can design and analyze the filter output effectively, precisely, easily with the help of Simulink in MATLAB. It is more practical and adjustable at any time by contrasting characteristics of the filter, in order to achieve the optimal design of the filter. Judging the output spectrum of mixed signal the filter design is achieved with the given specified parameters to fulfill the designer requirement. Further we observed that the side lobe attenuation decreases as the filter order increases, resulting the better performance of the filter which leads to fulfill the requirement.

\section{REFERENCES}

[1]. Cetin A E, Gerek O N, Yardimci Y, "Equiripple FIR filter design by the FFT algorithm," Signal Processing Magazine, 1997,14(2), pp. 60-64.

[2]. Yagyu M, Yoshida T, Nishihara A, "Design of FIR Digital Filters with Minimum Weight Representation," IEEE Transactions on Circuits and Systems, 1995, 40(3), pp. 227230.

[3]. Bougas P, Kalivas P, "Pipelined array-based FIR filter folding," IEEE Transactions on Circuits and Systems, 2005, 52(1), pp. 108-118.

[4]. Gang G Y, Qing Z, Yuanjin L, "Discrete-time Approximation of Filters," Journal of Control Theory and Applications, 2004, pp 1-10.

[5]. Jongsun P, Muhammad K, Roy K, "High-performance FIR filter design based on sharing multiplication," IEEE Transactions on VLSI, 2003, 11(2), pp. 244-253.

[6]. Cheng C, Parhi K K, "Low-cast Parallel FIR Filter Structures with 2- stage Parallelism," IEEE Transactions on Circuits and Systems, 2007, 54(2), pp. 280-290.

[7]. Hermanowicz E, Johansson H, "A Complex Variable Fractional-Delay FIR filter Structure," IEEE Transactions on Circuits and Systems II, 2007, 54(9), pp 785-789.

[8]. J. Proakis and D. G. Manolakis, "Digital Signal Processing" fourth edition, Prentice-Hall, 2007

[9]. Emmanuel C. Ifeachor and Barrie W. Jervis, "Digital Signal Processing"second edition, Pearson Education Limited, 2002

[10]. Shengli Yan, "FIR filter principle and design method," Changchun Engineering college journal, 2003, 4(1), pp 63-65.

[11]. Kaiyu Xu, Zhongming Zhu, Yaling Lu, "Digital signal processing," Electronics and industry press, 2005, pp. 137138.

[12]. Zhenhe Sun, Wei Yu, Chen He, "FIR digital filter design and MATLAB simulation", 2012 International Conference on Measurement, Information and Control (MIC) pp 677 - 680 